\title{
Professional Opera Tenors' Vocal Tract Configurations in Registers
}

\author{
Matthias Echternach ${ }^{a}$ Johan Sundberg ${ }^{c}$ Michael Markl ${ }^{b}$ Bernhard Richter ${ }^{a}$ \\ a Department of Musicians' Medicine and 'bepartment of Clinical Radiology, Medical Physics, \\ Freiburg University Medical Center, Freiburg, Germany; ' Department of Speech, Music and Hearing, \\ Royal Technical University, Stockholm, Sweden
}

\section{Key Words}

Magnetic resonance imaging $\cdot$ Voice $\cdot$ Register . Articulation

\begin{abstract}
Objective: Tenor singers may reach their top pitch range either by shifting from modal to falsetto register or by using their so-called 'voix mixte'. Material and Methods: In this study, dynamic real-time MRI of 8 frames per second was used to analyze the vocal tract profile in 10 professional opera tenors, who sang an ascending scale from $\mathrm{C} 4(262 \mathrm{~Hz})$ to A4 $(440 \mathrm{~Hz})$ on the vowel /a/. The scale included their register transition and the singers applied both register techniques in different takes. Results: Modal to falsetto register changes were associated with only minor vocal tract modifications, including elevation and tilting of the larynx and a lifted tongue dorsum. Transitions to voix mixte, by contrast, were associated with major vocal tract modifications. Under these conditions, the subjects widened their pharynges, their lip and jaw openings, and increased their jaw protrusion. These modifications were stronger in more 'heavy' tenors than in more 'light' tenors. The acoustic consequences of these articulatory changes are discussed.
\end{abstract}

Copyright ๑ 2010 S. Karger AG, Basel

\section{Introduction}

Vocal registers and their origins have been the subject of extensive research and controversy for more than 200 years [1-6]. Already in the 19th century Müller [7] concluded, by means of experiments on an excised human larynx, that vocal registers are dependent on the tension of the vocal folds. Later, van den Berg and Tan [1] found that in falsetto the activity of the cricothyroid muscle was dominating while in modal register the vocalis muscle dominated. Hirano et al. [8] found further support for these findings in EMG data.

Contraction of the cricothyroid muscle will change the angle between the thyroid and cricoid cartilages. This implies that this angle should differ between the falsetto register with its dominating cricothyroid muscle activity as compared to the modal register with its dominating vocalis contraction. However, the variation of the angle between these cartilages under conditions of register function has not been previously studied.

Presented in part at the 6th International Conference on Voice Physiology and Biomechanics (ICVPB), Tampere, Finland, August 6-9, 2008.

\section{KARGER \\ Fax +41613061234 E-Mail karger@karger.ch} www.karger.com
Matthias Echternach, MD

Institute of Musicians' Medicine, Freiburg University Medical Center

Breisacher Strasse 60, DE-79106 Freiburg (Germany)

Tel. +49761270 6161, Fax +497612706169

E-Mail matthias.echternach@uniklinik-freiburg.de 
Table 1. Subjects with age, current repertoire and classification according to Bunch and Chapman [19]

\begin{tabular}{ccll}
\hline Subject & Age & Current repertoire & Classification \\
\hline 1 & 34 & Werther (Werther, Massenet), Rudolfo (Bohème, Puccini) & national \\
2 & 30 & Tamino (Zauberflöte, Mozart), Idamante (Idomeneo, Mozart) & national \\
3 & 37 & Goro (Butterfly, Puccini), Mime (Rheingold and Siegfried, Wagner) & national \\
4 & 31 & Tamino (Zauberflöte, Mozart), Aufidio (Lucio Silia, Mozart) & national \\
5 & 37 & Bucklaw (Lucia, Donizetti), Loge (Rheingold, Wagner) & national \\
6 & 25 & Varo (Ezio, Gluck), Nencio (L'infedelta delusa, Haydn) & international \\
7 & 32 & Siegmund (Walküre, Wagner), Froh (Rheingold, Wagner) & international \\
8 & 45 & Cavaradossi (Tosca, Puccini), Pinkerton (Butterfly, Puccini) & national \\
9 & 30 & Monostratos (Zauberflöte, Mozart) & international \\
10 & 43 & Tito (Tito, Mozart), Tamino (Zauberflöte, Mozart) & \\
\hline
\end{tabular}

Sundberg and Högset [9] analyzed the voice source in professional singers and found systematic differences between these registers. It is commonly assumed that subglottal and vocal tract resonance influences registers and register transitions. The spectral changes associated with register shifts have been assumed to reflect vocal tract changes $[10,11]$. Also, model experiments have suggested that the vocal tract interacts with the voice source [12-14] and thus may have an influence on vocal registers.

Vocal tract resonances are mainly determined by the shape of the vocal tract. Hence, if vocal tract resonance is relevant to register, vocal tract shape should change depending on register function. However, so far, only few studies have focused on vocal tract shape in register transitions. Using conventional X-ray technology, Luchsinger [15] demonstrated in 1949 that the configuration of the laryngeal ventricle differs between modal and falsetto registers. Using computer tomography, Tom et al. [16] examined vocal tract configurations in three dimensions during speech in modal register and during singing in falsetto register. However, phonation frequency differed by one octave between these two conditions and no register transition was examined.

In two previous pilot studies we applied dynamic realtime MRI for analyzing vocal tract changes during register transitions $[17,18]$. In one of these studies 2 professional male singers, a tenor and a baritone, served as subjects. We found minor changes when these singers shifted from modal to falsetto [18], but marked changes when they avoided a register shift. In the latter condition, the baritone raised his tongue dorsum, widened his jaw opening, and retracted his lower jaw while the tenor lifted his uvula. Both subjects widened their lip opening and their oropharynx.

Tenors' Vocal Tract Configurations in Registers
During our pilot studies $[17,18]$ the laryngeal structures were too blurred to show reliable landmarks for measurement of laryngeal distances and angles. Since then, MRI technology has been further developed. Now, realtime MRI recordings can be performed at 8 frames per second with acceptable resolution of laryngeal structures.

The present study aimed to analyze whether there are common patterns of vocal tract modifications associated with register functions and register transitions in professional opera tenors. The second goal was to analyze if tenors change their cricothyroid angle when they shift register from modal to falsetto.

\section{Material and Methods}

Ten professional opera tenors served as subjects. Table 1 shows their age, current repertoire and classification according to the Bunch and Chapman [19] criteria. These criteria describe performance abilities ranging from an international level (superstar) to local community and amateur singers with comprehensive subcategories. At the time of recording, none of the subjects complained of any vocal symptoms. Vocal function pathologies were excluded by history, auditory and videostroboscopic examinations.

All subjects were examined radiologically with a 3.0-tesla TIM TRIO (Siemens, Germany) MRI device as described elsewhere [17, $18,20]$. Real-time MRI was performed with a temporal resolution of 8 images per second. The images showed one sagittal slab of $11 \mathrm{~mm}$ thickness using an rf-spoiled $2 \mathrm{D}$ GRE sequence $\left(\mathrm{G}_{\max }=\right.$ $40 \mathrm{mT} / \mathrm{m}$, rise time $=200 \mathrm{~ms}$, standard 12-channel head and neck coils) with the following sequence parameters: spatial resolution $=1.4 \times 2.2 \mathrm{~mm}^{2}$, TE $0.84 \mathrm{~ms}$, TR $2.53 \mathrm{~ms}$, FA $5^{\circ}$, bandwidth $650 \mathrm{~Hz} / \mathrm{Px}$, matrix $192 \times 144$, FOV $250 \times 215 \mathrm{~mm}^{2}$, GRAPPA $=$ 2 . Also the audio signal was recorded by means of an optic microphone (MR confon, Magdeburg, Germany; OptiMRI Noise Reduction Software, Optoacoustics Ltd., Or-Yehuda, Israel). Like in our previous studies the subjects were provided with the audio signal over headphones (MR confon) as an acoustic foldback. 
All subjects sang an ascending diatonic major scale from C4 $(262 \mathrm{~Hz})$ to A4 $(440 \mathrm{~Hz})$, starting in the modal register. In the first condition the subjects were asked to perform a register shift between E4 and F4, while in the second condition, they sang the same scale as they would do on stage. Henceforth the register they used above F4 will be referred to as voix mixte. The pitch transition E4 to F4 for a register shift was chosen since - according to our experience - professional tenors often regard this pitch range as the typical passaggio region. The vowel /a/ was chosen for all sequences so as to avoid the articulatory effects that can be expected when the fundamental frequency exceeds the normal value of the first formant.

Since other methods for detection of register transitions, i.e. electroglottography or high-speed imaging, cannot be used inside the MRI device, register transitions were identified perceptually only. During the recording 3 experts checked that the subjects produced the desired register conditions. Also, directly after each sequence, the subjects were asked if they felt that they had produced the register conditions asked for. Only sequences found acceptable by all 3 experts as well as by the singer himself were subsequently analyzed.

In each frame of the MRI material a series of measures were taken (fig. 1): (a) distance between the lips, (b) jaw opening, defined as the distance between the spina of the upper jaw and the lower front edge of the mandible, (c) tongue dorsum, defined as the maximum distance from a line touching the lower contour of the mandible and up to the highest cranial point of the tongue contour, (d) the distance between the lower front edge of the mandible and the mucosal cover of the spine at a 90-degree angle, (e) pharynx width measured as the shortest distance between the posterior contour of the tongue, and the mucosal cover of the spine, and (f) the distance between a line extending the upper contour of the hard palate and a parallel line tangent to the lowermost part of the uvula contour. An auxiliary line (A) was drawn for the measurement of the larynx position and laryngeal tilting. This auxiliary line connects the cranial-most part of the dens axis and the caudo-anterior edge of the sixth vertebra. The larynx position was measured (g) at the distance from the cranial-most part of the dens axis to the point where auxiliary line A crosses a line (auxiliary line B) from the anterior commissure rectangular to auxiliary line A, and (h) the larynx tilt measured as the angle between auxiliary line A and a line from the anterior commissure to the vocal process.

In the audio recording, the fundamental frequency was identified by means of the PRAAT software (University of Amsterdam, The Netherlands). The relationship between fundamental frequency, register condition and each of the MRI measures was analyzed. Statistical nonparametric analysis was carried out by means of SPSS 15.0 software using Friedman, Wilcoxon, and Mauchly tests. In addition, Pearson correlation was determined for MRI data. The general level of significance was set at $\alpha=0.05$.

\section{Results}

The 3 experts agreed that in all subjects, except subject 10, falsetto was perceptually different from the voix mixte, and sequences ending in voix mixte were clearly louder that than those ending in falsetto.

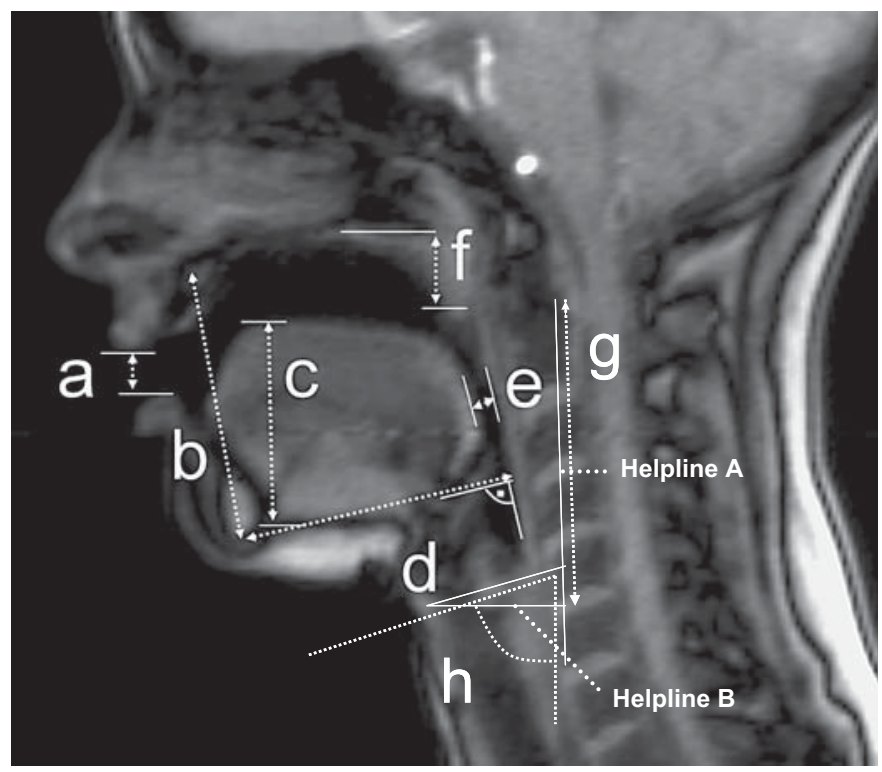

Fig. 1. Auxiliary lines and distances measured in MR images: $\mathrm{a}=$ lip opening, $\mathrm{b}=$ jaw opening, $\mathrm{c}=$ tongue dorsum, $\mathrm{d}=$ jaw protrusion, $\mathrm{e}=$ oropharynx width, $\mathrm{f}=$ uvula elevation, $\mathrm{g}=$ larynx position, and $\mathrm{h}=$ angle of larynx tilt (see text).

The MR profiles showed clear modifications of vocal tract shape in most subjects' transitions to voix mixte: the lip and jaw openings were widened, the jaw protruded, pharynx width increased and the uvula was elevated (fig. 2a-f). By contrast, transitions to falsetto register were associated with minor changes only: mainly, the larynx was elevated and tilted (fig. $2 g, h$ ). In addition to increased lip opening, 3 of the subjects protruded their lips in the voix mixte register (fig. 3).

The measures from the MRI material for all tones produced in modal register (C4-E4) and all tones produced in voix mixte or falsetto (F4 to A4) were pooled and averaged, and the results were analyzed statistically (fig. 4, 5). Table 2 shows the correlations between each pair of measures.

Lip opening was significantly correlated with jaw opening, as expected, and the trendlines for the falsetto and for the voix mixte were nearly parallel (fig. 6). The slope constants revealed that lip opening was much less than jaw opening. Moreover, the intercept was much smaller in falsetto, which indicates that the subjects actively reduced their lip opening more in falsetto than in voix mixte (fig. 6). Also, widening of the lip opening was associated with a retraction of the jaw. As expected, the pharynx width was correlated with the jaw protrusion. 

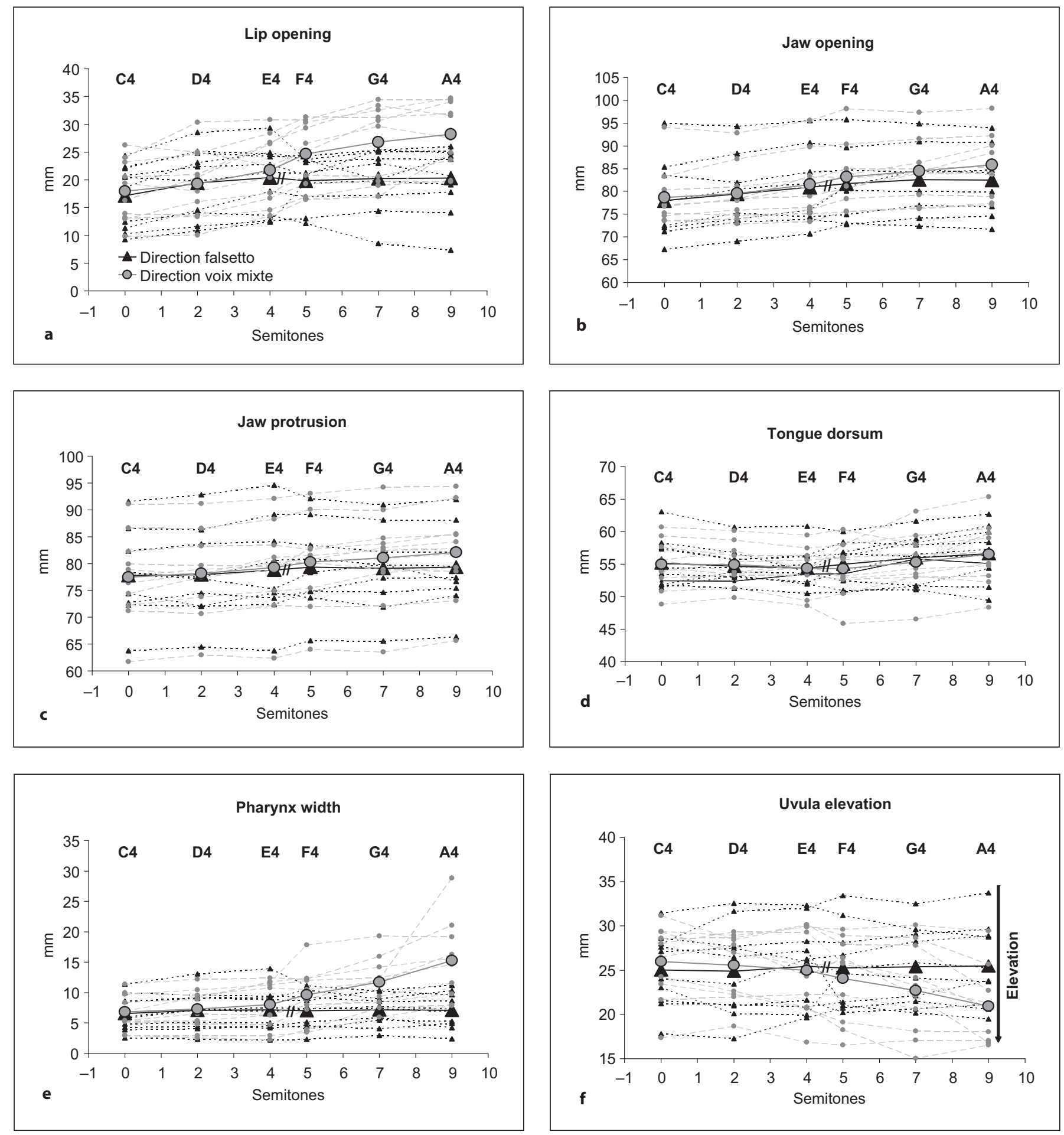

Fig. 2. Distances measured in MR images for sequences to falsetto (black) and to voix mixte (gray): a lips, b jaw opening, $\mathbf{c}$ tongue dorsum, $\mathbf{d}$ jaw protrusion, e oropharynx width, $\mathbf{f}$ uvula elevation. Dashed lines refer to subjects, solid lines and bold symbols represent mean values across subjects. The double slash represents the register shift from modal to falsetto register. 

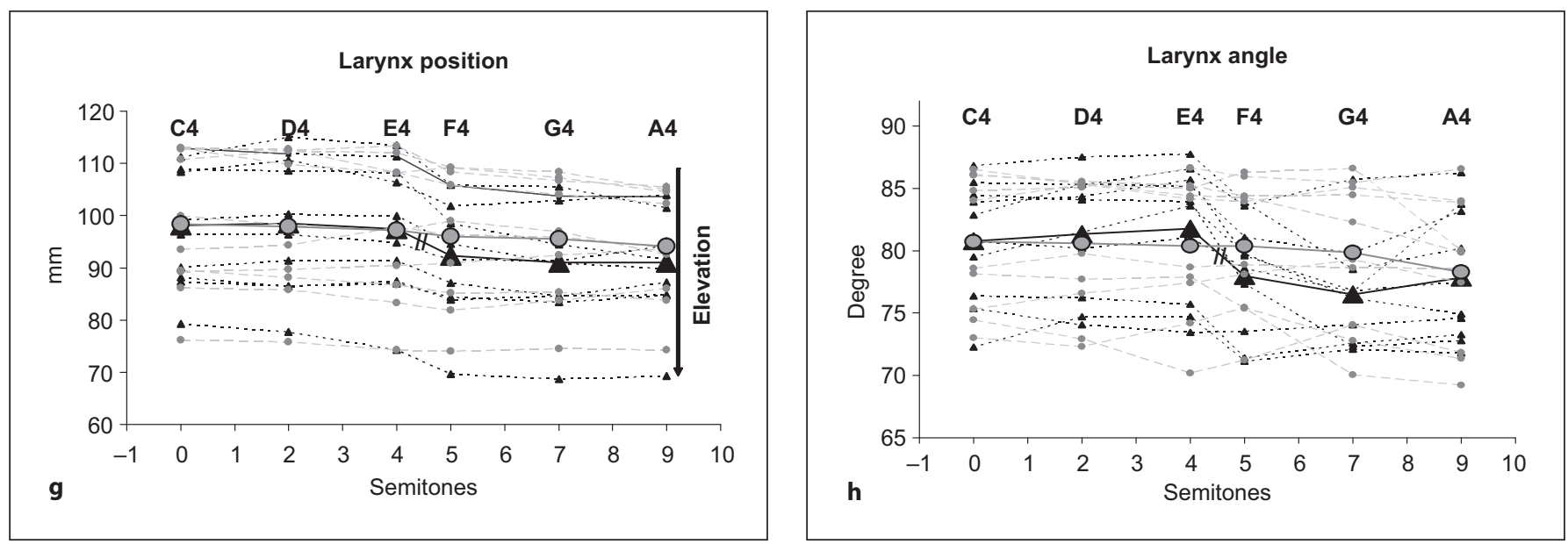

Fig. 2. Distances measured in MR images for sequences to falsetto (black) and to voix mixte (gray): $\mathbf{g}$ larynx position, and $\mathbf{h}$ larynx tilt, defined as shown in figure 1. Dashed lines refer to subjects, solid lines and bold symbols represent mean values across subjects. The double slash represents the register shift from modal to falsetto register.

Fig. 3. Images of a tenor (subject 1) singing D4 and G4 (left and right panels) for sequences going into falsetto and to voix mixte (upper and lower panels, respectively).

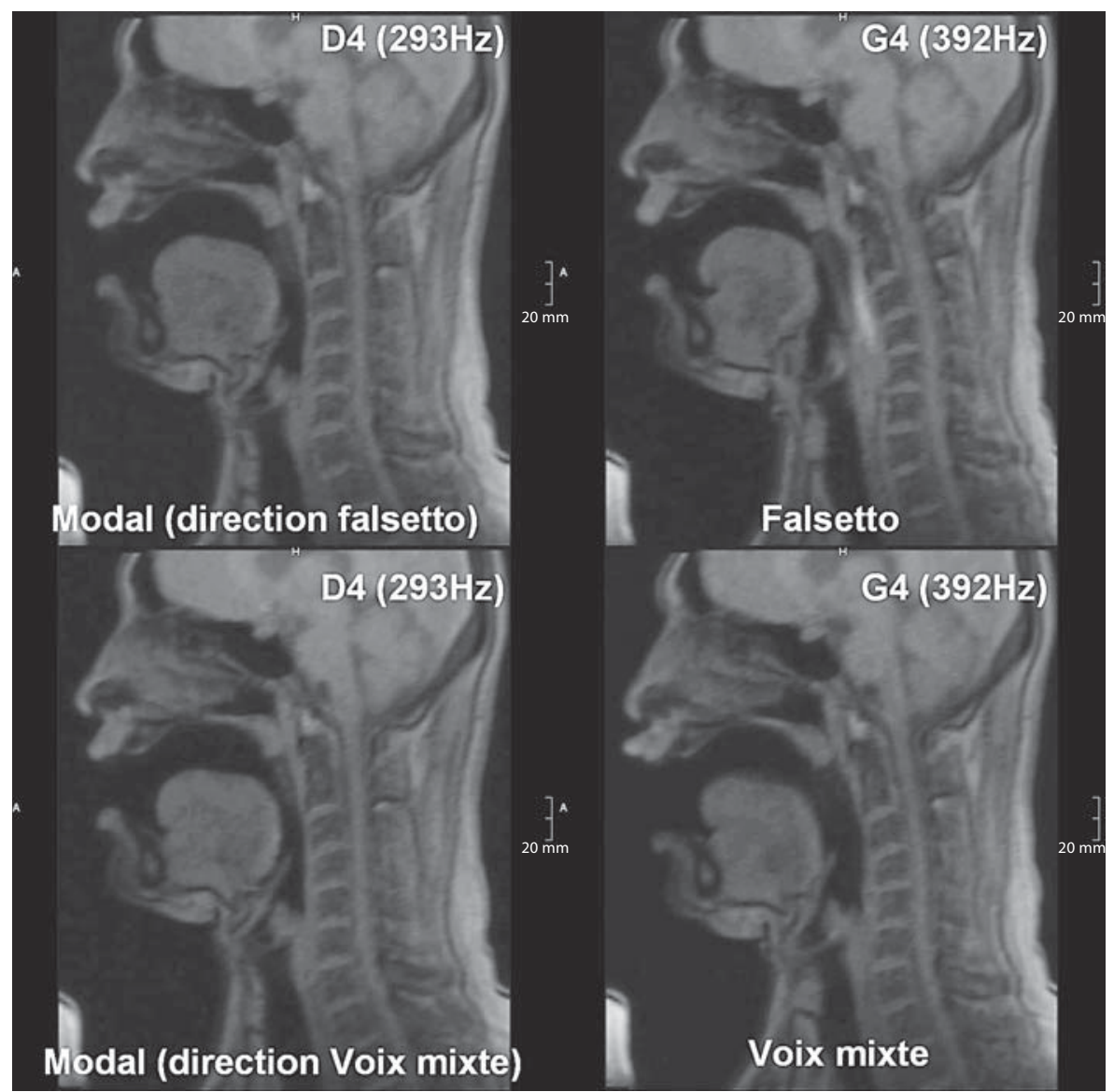

Echternach/Sundberg/Markl/Richter 


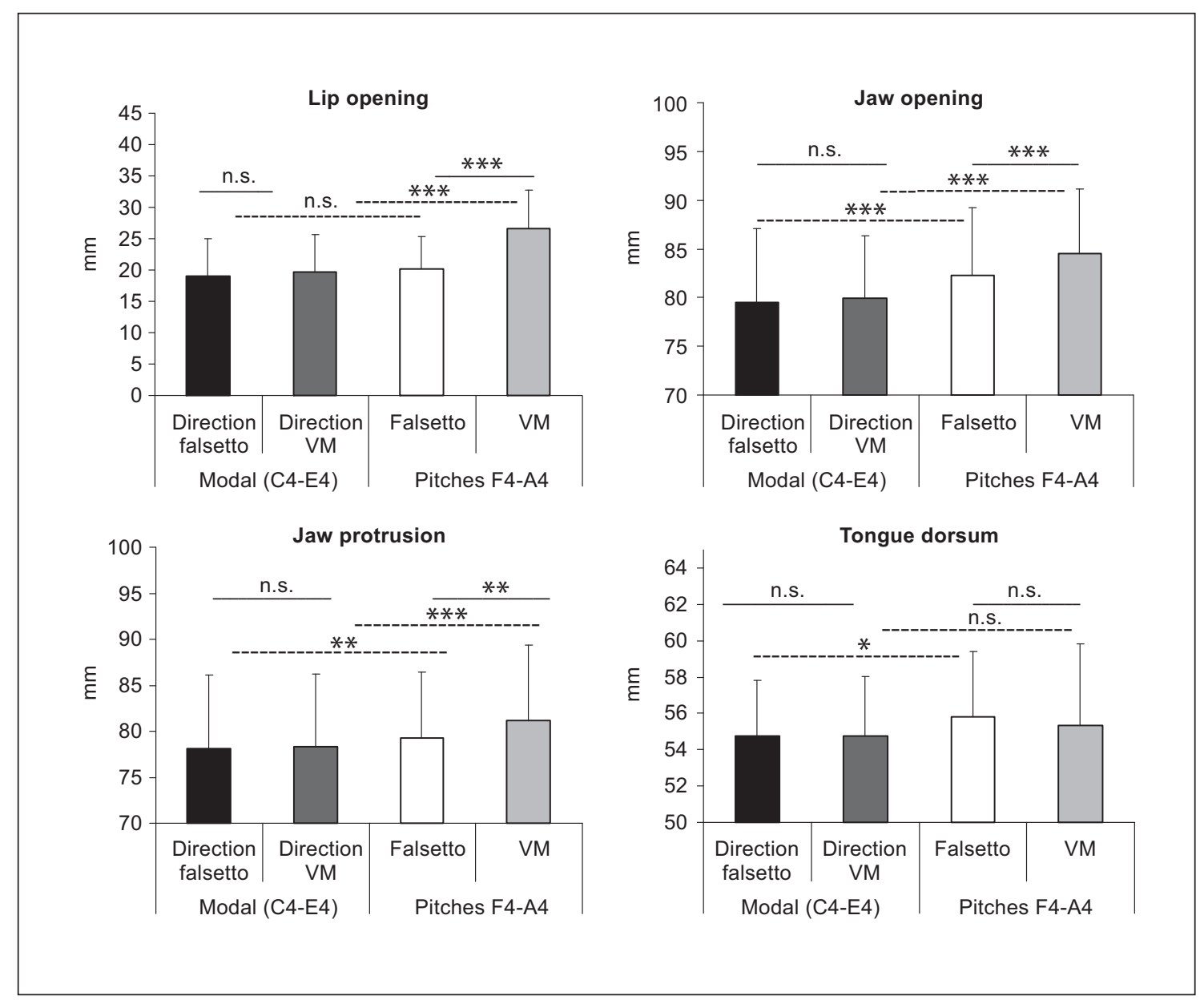

Fig. 4. Mean values and standard deviations across subjects and indicated pitches for lip and jaw opening, jaw protrusion and tongue dorsum for the indicated conditions. $\mathrm{VM}=$ Voix mixte; statistical significance: ${ }^{*} \mathrm{p}<$ $0.05,{ }^{* *} \mathrm{p}<0.01,{ }^{* * *} \mathrm{p}<0.001$, n.s. $=$ nonsignificant. Error bars represent one standard deviation.

Elevation of the larynx was associated with a more tilted larynx. In addition both larynx tilt and larynx elevation were highly correlated with jaw protrusion. When the uvula was elevated, the height of the tongue dorsum decreased.

For most of the distance data, the Mauchly test of sphericity indicated that there were strong differences between the subjects. As the different subjects showed strikingly different articulatory behavior we show the raw data as seen in figure 2. Yet, all subjects widened lip opening and elevated the uvula in the voix mixte condition. In both conditions, the tongue dorsum showed no systematic differences for all subjects.

To illustrate the range of modifications of the vocal tract in different tenors, figure 7 presents MRI profiles of a light/lyrical tenor (subject 10) and a more heavy helden- tenor voice (subject 7). The images were taken from the sequences from modal to falsetto (fig. $7 \mathrm{a}, \mathrm{b}$ ) and from modal to voix mixte (fig. 7c, d). Both these subjects perform internationally. The modifications were clearly stronger for the heavier voice (subject 7) at high pitch.

\section{Discussion}

This investigation presents data on vocal tract modifications associated with a shift of vocal register in a group of 10 professional opera tenors. The results basically confirm the observation made in our previous pilot study, that minor vocal tract changes occurred in few parameters only, when the singers shifted register from modal to falsetto [18], while several and substantial mod- 


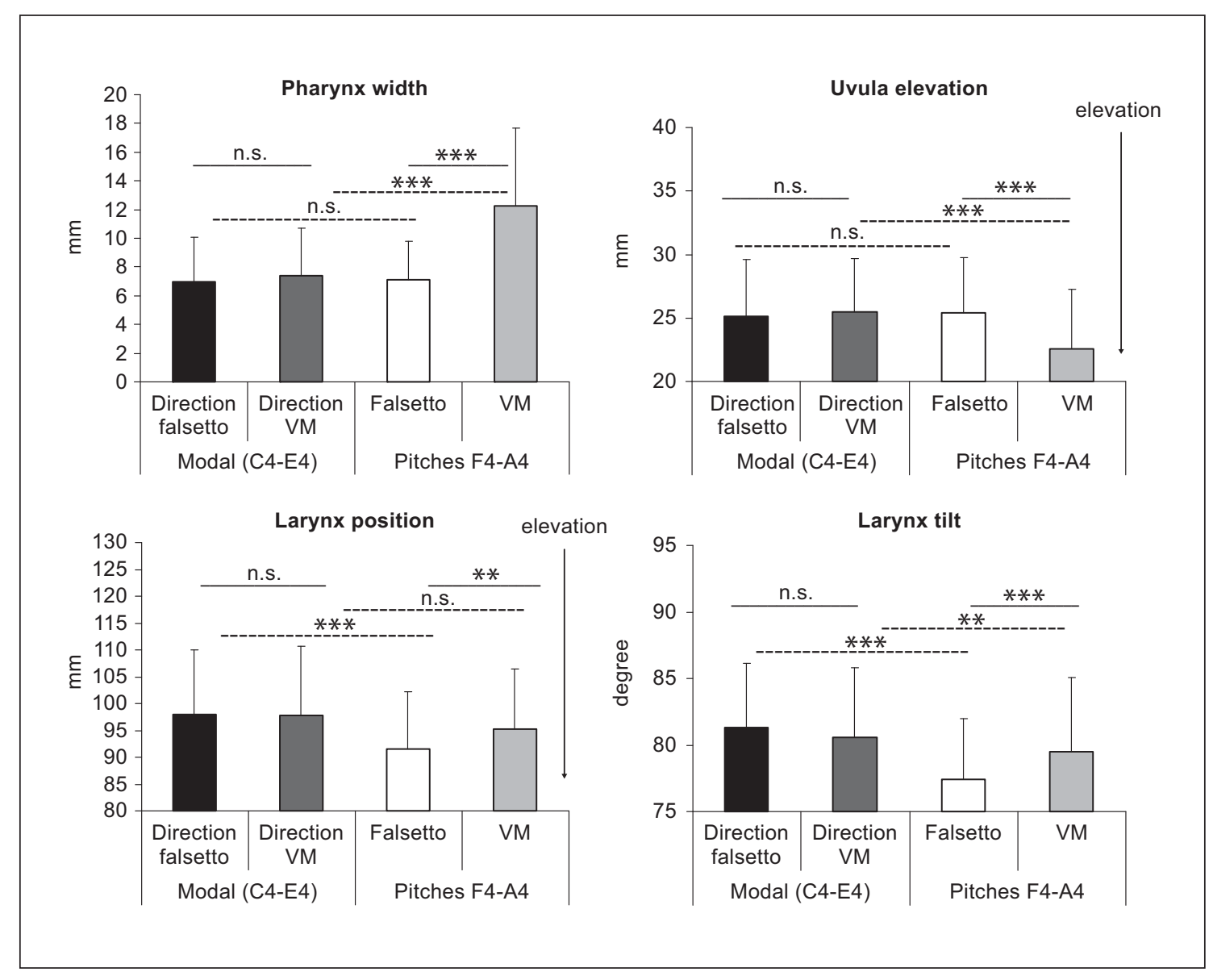

Fig. 5. Mean values and standard deviations across subjects and indicated pitch for pharynx width, uvula elevation, larynx position and larynx angle for the indicated conditions. VM $=$ Voix mixte, ${ }^{* *} \mathrm{p}<0.01,{ }^{* *} \mathrm{p}<0.001$, n.s. = nonsignificant. Error bars represent one standard deviation.

ifications were found when the singers ended a scale in voix mixte. However, our present data also reveal considerable individual variability. Major vocal tract changes were seen in the heldentenor (subject 7).

Phonation at high pitch is one of the most important issues in the education of professional tenor voices. At fundamental frequency, near E4 $(330 \mathrm{~Hz})$ the modal register is limited in its frequency expansion and singers usually have to decide whether or not they should change the register to falsetto or continue in the modal register in a modulated way. The latter solution is often referred to by the term voix mixte. Since falsetto is normally associated with fewer and weaker overtones [9], switching to falsetto does not appear as an attractive solution in stage performances for most tenors. In our pilot study we found substantial vocal tract modifications accompany- ing a transition to voix mixte in a professional tenor [18]. Such modifications were found also in most subjects analyzed in the present investigation.

All modifications of vocal tract shape, e.g. the observed elevation of the uvula and widening of the pharynx, will affect the formant frequencies and hence the acoustic reactance encountered by the voice source. This may change the conditions for vocal fold vibration [13, 14]. Both lip and jaw opening were wider in voix mixte than in modal voice, and this should raise both the first and the second formants. In some subjects, however, the lips were found to be more protruded in voix mixte, which will tend to counteract the acoustic effect of increased lip opening. In falsetto the larynx was higher than in modal voice. This will tend to increase formant frequencies. At the same time the singers tended to sing 
Fig. 6. Relationship between lip opening and jaw opening. Dashed and solid trendlines refer to falsetto and voix mixte, respectively. The equation refers to the slope of the regression line.

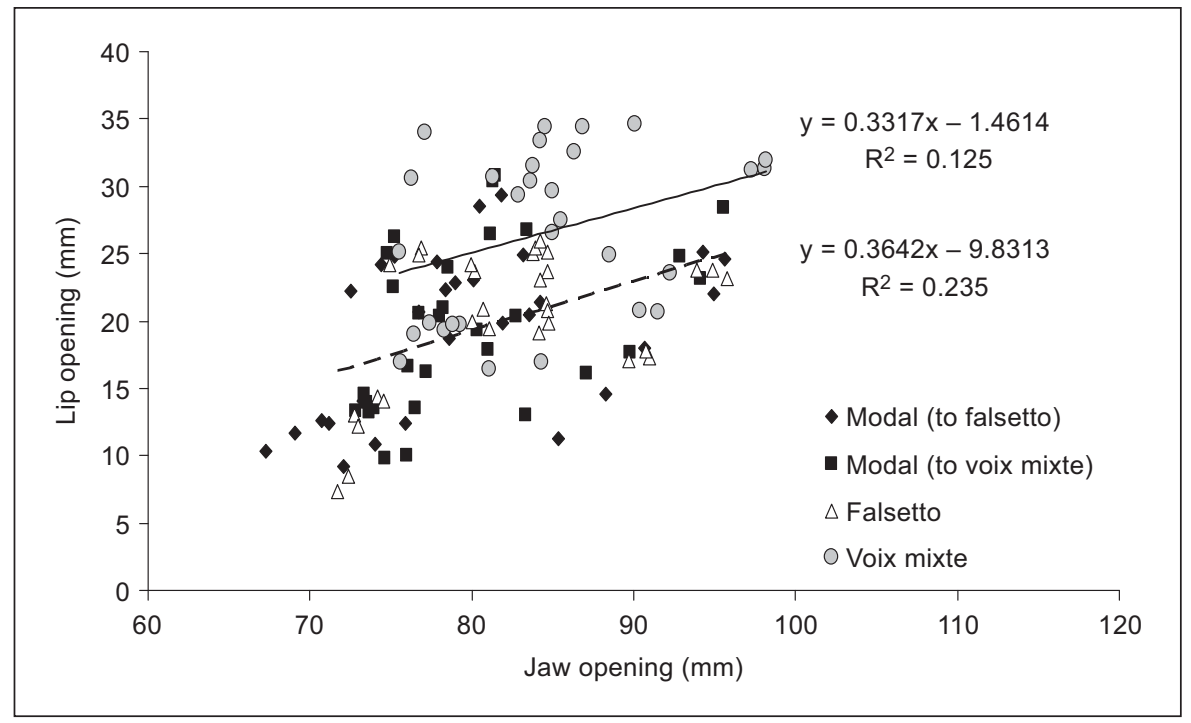

Table 2. Pearson correlation coefficient ( $\mathrm{r}$ ) and $\mathrm{p}$ values corrected for multiple comparisons for all vocal tract distance measures, independent of register condition

\begin{tabular}{|c|c|c|c|c|c|c|c|c|c|}
\hline & & LO & $\mathrm{JO}$ & JP & TD & PW & UE & LE & $\mathrm{LT}$ \\
\hline \multirow[t]{2}{*}{ LO } & $\mathrm{r}$ & 1.00 & 0.49 & -0.19 & 0.00 & 0.29 & 0.26 & 0.16 & 0.46 \\
\hline & $\mathrm{p}$ value & & 0.00 & 0.04 & 0.99 & 0.00 & 0.00 & 0.09 & 0.00 \\
\hline \multirow[t]{2}{*}{$\mathrm{JO}$} & $\mathrm{r}$ & 0.49 & 1.00 & 0.13 & 0.69 & 0.15 & -0.24 & -0.23 & 0.02 \\
\hline & $\mathrm{p}$ value & 0.00 & & 0.16 & 0.00 & 0.10 & 0.01 & 0.01 & 0.79 \\
\hline \multirow[t]{2}{*}{$\overline{\mathrm{JP}}$} & $\mathrm{r}$ & -0.19 & 0.13 & 1.00 & -0.04 & 0.41 & 0.17 & -0.58 & -0.60 \\
\hline & $\mathrm{p}$ value & 0.04 & 0.16 & & 0.64 & 0.00 & 0.07 & 0.00 & 0.00 \\
\hline \multirow[t]{2}{*}{$\overline{\mathrm{TD}}$} & $\mathrm{r}$ & 0.00 & 0.69 & -0.04 & 1.00 & 0.03 & -0.49 & -0.19 & -0.22 \\
\hline & $\mathrm{p}$ value & 0.99 & 0.00 & 0.64 & & 0.78 & 0.00 & 0.03 & 0.02 \\
\hline \multirow[t]{2}{*}{$\overline{\mathrm{PW}}$} & $\mathrm{r}$ & 0.29 & 0.15 & 0.41 & 0.03 & 1.00 & 0.11 & -0.05 & -0.38 \\
\hline & $p$ value & 0.00 & 0.10 & 0.00 & 0.78 & & 0.25 & 0.62 & 0.00 \\
\hline \multirow[t]{2}{*}{$\overline{\mathrm{UE}}$} & $\mathrm{r}$ & 0.26 & -0.24 & 0.17 & -0.49 & 0.11 & 1.00 & -0.12 & 0.15 \\
\hline & $\mathrm{p}$ value & 0.00 & 0.01 & 0.07 & 0.00 & 0.25 & & 0.19 & 0.11 \\
\hline \multirow[t]{2}{*}{$\overline{\mathrm{LE}}$} & $\mathrm{r}$ & 0.16 & -0.23 & -0.58 & -0.19 & -0.05 & -0.12 & 1.00 & 0.38 \\
\hline & $\mathrm{p}$ value & 0.09 & 0.01 & 0.00 & 0.03 & 0.62 & 0.19 & & 0.00 \\
\hline \multirow[t]{2}{*}{$\overline{\mathrm{LT}}$} & $\mathrm{r}$ & 0.46 & 0.02 & -0.60 & -0.22 & -0.38 & 0.15 & 0.38 & 1.00 \\
\hline & $\mathrm{p}$ value & 0.00 & 0.79 & 0.00 & 0.02 & 0.00 & 0.11 & 0.00 & \\
\hline
\end{tabular}

LO = Lip opening; JO = jaw opening; JP = jaw protrusion; TD = tongue dorsum; $\mathrm{PW}$ = pharynx width; $\mathrm{UE}=$ uvula elevation; $\mathrm{LP}=$ larynx position; $\mathrm{LT}=$ larynx tilt.

with a smaller lip opening in falsetto than in voix mixte, thus reducing or counteracting the effect on the formant frequencies of the higher larynx.

Some authors independently considered a 'covered' voice ('gedecktes Singen', 'voix sombrée') as helpful for phonation at high pitch, a technique that includes lowering of the larynx and widening of the pharyngeal and supraglottic areas $[3,21]$. In the present study similar observations were made in voix mixte as in falsetto. Van Deinse [21] also claimed that it was helpful to relax the 

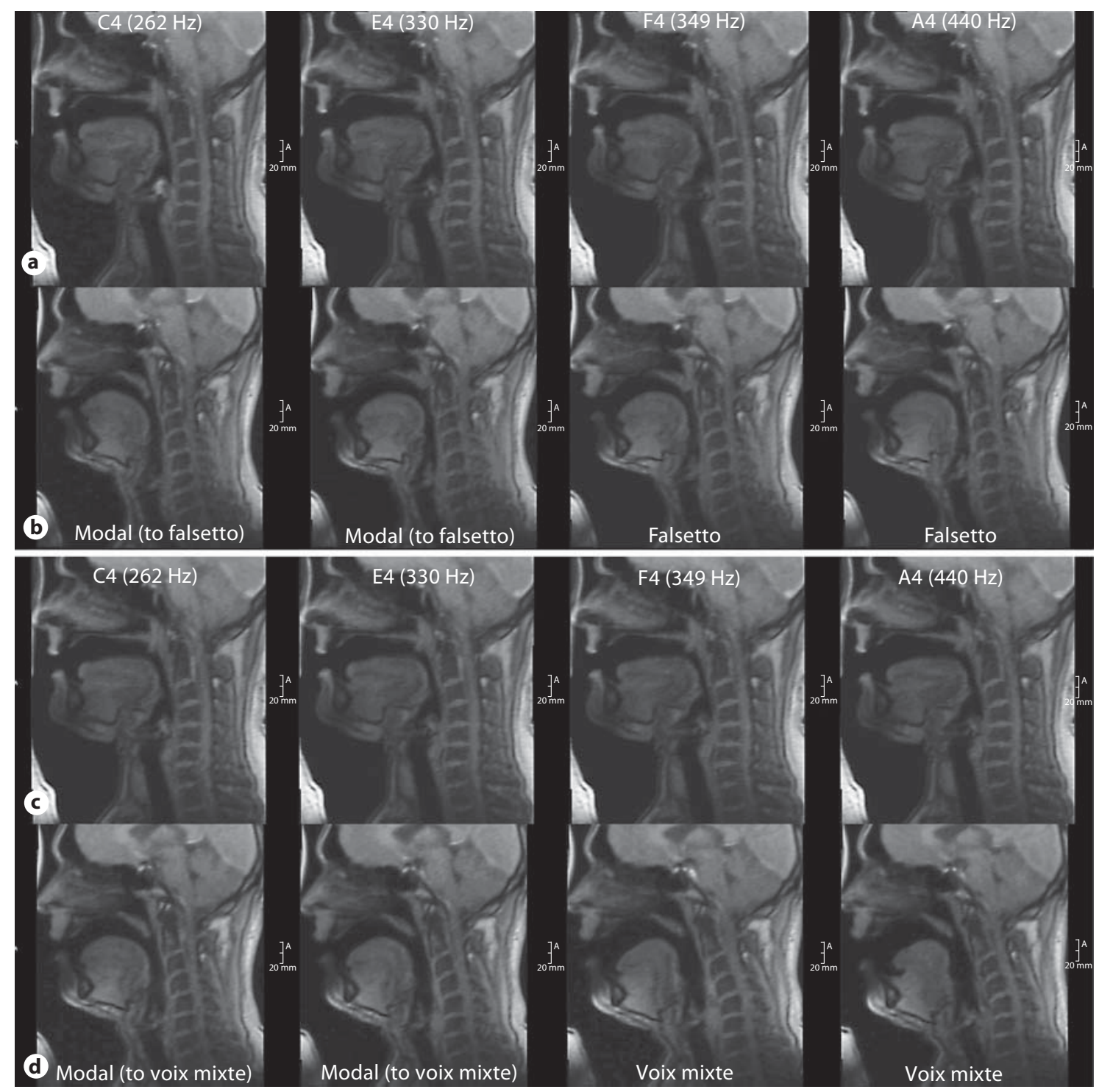

Fig. 7. Images of a lyrical tenor $(\mathbf{a}, \mathbf{c})$ and a heldentenor $(\mathbf{b}, \mathbf{d})$, from sequences with direction falsetto and voix mixte, respectively.

uvula at high pitch, but our data failed to corroborate this claim. Detailed volumetric and acoustic analyses may elucidate more in detail the relevance of nasal coupling in singing.

In previous investigations it was shown that the falsetto register is associated with a more forceful contraction of the cricothyroid muscle than in the vocalis muscle [22]. Activation of the cricothyroid muscle leads to an approximation of the anterior parts of the thyroid and cricoid cartilages. Our observation that the larynx was found to be more tilted in falsetto register seems to reflect this same mechanism.

It seems relevant to mention that different kinds of tenors were included in this study, from very light tenors, who mainly perform in concert halls (subject 10), to heldentenor (subject 7). It should be kept in mind that these two tenor 'Fachs' sing quite different repertoires and face quite different demands on voice production. It is therefore tempting to speculate about the impact of 'Fach' on our results. At high pitch, we found greater vocal tract 
differences between the falsetto and the voix mixte registers when sung by 'heavy' Fach tenors, such as the heldentenor, than when sung by 'light' Fach tenors. In our study, the heldentenor (subject 7) was found to curve his neck at high pitch (fig. 7d). As a consequence his head was tilted forward. In the previous investigation, the same observation was made for the baritone when he kept his modal register throughout his passaggio region [18]. This might account for the sometimes striking similarities in timbre in the high range of heldentenors and baritones. In our 'light' tenor (subject 10) we did not find any major vocal tract shape differences between his modal and falsetto or voix mixte registers. Clearly, systematic studies are needed to investigate the relevance of Fach to vocal tract shaping.

\section{Conclusions}

Change of register from modal to falsetto was associated with only minor vocal tract modifications in professional tenors. These changes included an elevation of the larynx and a raised tongue dorsum. Additionally, greater thyroid tilt seems to be consistent with the idea of a dominance of cricothyroid muscle contraction in falsetto. Transition over the passaggio region to voix mixte, by contrast, was associated with strong vocal tract modifications. Here, the subjects widened their pharynges, their lip and jaw openings, and increased their jaw protrusion. These modifications were greater in 'heavier' than in 'light' tenors.

\section{References}

1 Van den Berg JW, Tan TS: Results of experiments with human larynxes. Pract Otorhinolaryngol (Basel) 1959;21:425-450.

2 Nadoleczny M: Untersuchungen über den Kunstgesang. Berlin, Springer, 1923.

3 Large J: Towards an integrated physiologicacoustic theory of vocal registers. NATS Bull. 1972;28:18-36.

4 Hollien $\mathrm{H}$ : On vocal registers. J Phonet 1974; 2:125-143.

5 Henrich N: Mirroring the voice from Garcia to the present day: some insights into singing voice registers. Logoped Phoniatr Vocol 2006;31:3-14

6 Garcia M: Mémoire sur la voix humaine présenté à l'Académie des Sciences en 1840, ed 2. Paris, Duverger, 1847.

7 Müller J: Handbuch der Physiologie des Menschen für Vorlesungen. Koblenz, Hölscher, 1840.

8 Hirano M, Vennard W, Ohala J: Regulation of register, pitch and intensity of voice: an electromyographic investigation of intrinsic laryngeal muscles. Folia Phoniatr (Basel) 1970;22:1-20
-9 Sundberg J, Högset C: Voice source differences between falsetto and modal registers in counter tenors, tenors and baritons. Logoped Phoniatr Vocol 2001;26:26-36.

10 Miller DG: Registers in Singing: Empirical and Systematic Studies in the Theory of the Singing Voice. Groningen, University of Groningen, 2000.

-11 Neumann K, Schunda P, Hoth S, Euler HA The interplay between glottis and vocal tract during the male passaggio. Folia Phoniatr Logop 2005;57:308-327.

12 Titze IR, Story BH: Acoustic interactions of the voice source with the lower vocal tract. J Acoust Soc Am 1997;101:2234-2243.

13 Titze IR, Riede T, Popolo P: Nonlinear source-filter coupling in phonation: vocal exercises. J Acoust Soc Am 2008;123:19021915.

14 Titze IR: Nonlinear source-filter coupling in phonation: theory. J Acoust Soc Am 2008; 123:2733-2749.

15 Luchsinger R: Falsett und Vollton der Kopfstimme. Arch Ohr Heilk 1949;155:505-519.

- 16 Tom K, Titze IR, Hoffman EA, Story BH: Three-dimensional vocal tract imaging and formant structure: varying vocal register, pitch, and loudness. J Acoust Soc Am 2001; 109:742-747.
17 Echternach M, Sundberg J, Arndt S, Markl $\mathrm{M}$, Schumacher M, Richter B: Vocal tract in female registers - a dynamic real-time MRI study. J Voice 2010;24:133-139.

18 Echternach M, Sundberg J, Arndt S, Breyer T, Markl M, Schumacher M, Richter B: Vocal tract and register changes analysed by real time MRI in male professional singers - a pilot study. Logoped Phoniatr Vocol 2008;33: 67-73.

19 Bunch M, Chapman J: Taxonomy of singers used as subjects in scientific research. J Voice 2000;14:363-369.

20 Breyer T, Echternach M, Arndt S, Richter B, Speck O, Schumacher M, Markl M: Dynamic MRI of swallowing and laryngeal motion using parallel imaging at 3T. Magn Reson Imaging 2009;27:48-54.

21 Van Deinse JB: Registers. Folia Phoniatr (Basel) $1981 ; 33: 37-50$

22 Van den Berg JW: Vocal ligaments versus registers. NATS Bull 1963;20:16-21. 